\title{
Ensaio pré-clínico com desfibrilador externo
}

\author{
Pre-clinical trial with external defibrillator
}

\author{
Amanda Assunção Vieira', Michele dos Santos Gomes da Rosa², Silvia Lemos Fagundes², Eduardo Mackmann ${ }^{3}$, \\ Francisco S. Inácio ${ }^{4}$, Stela Maria R. Krapf ${ }^{5}$, Giordano Cabral Gianotti ${ }^{6}$, Jefferson Braga Silva ${ }^{7}$, Thais Russomano ${ }^{8}$ \\ ${ }^{1}$ Farmacêutica, Mestre em Engenharia Elétrica/Biomédica (PUCRS), Centro de Microgravidade, PUCRS, Porto Alegre. \\ 2 Fisioterapeutas, Mestres em Engenharia Elétrica/Biomédica (PUCRS), Centro de Microgravidade, PUCRS, Porto Alegre. \\ ${ }^{3}$ Administrador, Mestre em Economia do Desenvolvimento (PUCRS), Toth Desenvolvimento Tecnológico LTDA, Tecnopuc, Porto Alegre. Lifemed Industrial \\ de Equipamentos e Artigos Médicos e Hospitalares S/A, Pelotas. \\ ${ }^{4}$ Engenheiro Eletricista, Toth Desenvolvimento Tecnológico LTDA, Tecnopuc, Porto Alegre. Lifemed Industrial de equipamentos e Artigos Médicos e Hospitalares S/A. \\ ${ }^{5}$ Farmacêutica-Bioquímica, Unidade de Bioquímica, Laboratório de Patologia, Hospital São Lucas, PUCRS, Porto Alegre. \\ ${ }^{6}$ Médico Veterinário, Doutor em Anestesiologia Animal, Centro de Modelos Biológicos Experimentais, PUCRS, Porto Alegre. \\ ${ }^{7}$ Médico, Professor Doutor do Departamento de Cirurgia da Faculdade de Medicina da PUCRS Doutor, Coordenador Laboratório de Habilidade Médicas \\ e Pesquisa Cirúrgica, FAMED, PUCRS, Porto Alegre. \\ ${ }^{8}$ Médica, Professora Doutora das Faculdades de Medicina e Ciências Aeronáuticas, Coordenadora do Centro de Microgravidade, PUCRS, Porto Alegre.
}

Este estudo recebeu apoio financeiro da Agência Brasileira da Inovação - Financiadora de Estudos e Projetos (FINEP).

\section{RESUMO}

Objetivos: Avaliar a eficácia da desfibrilação em resposta à fibrilação ventricular induzida e descrever as mudanças macroscópicas ocorridas nos corações de suínos após choque de descarga capacitiva exponencial truncada aplicado por protótipo de desfibrilador produzido no Brasil e por um desfibrilador de referência, líder de mercado internacional.

Métodos: Dezesseis suínos foram agrupados em quatro grupos de quatro animais, para verificar a eficácia da desfibrilação em resposta à fibrilação ventricular induzida, aplicando cargas de $50 \mathrm{~J}$ e $100 \mathrm{~J}$, assim como avaliar a segurança dos desfibriladores usados, através de análises macroscópicas da pele e do coração. Foram avaliados eritema, edema, bolhas, líquido na cavidade pericárdica, queimaduras e tecido carbonizado pós-choque.

Resultados: Todos os suínos, após um minuto da indução à fibrilação ventricular, desenvolveram taquicardia que progrediu à fibrilação ventricular. Quando utilizada baixa energia, o equipamento protótipo obteve $25 \%$ de sucesso com um suíno revertido, enquanto o equipamento referência obteve $50 \%$ de sucesso, com duas reversões. Entretanto, com energia a $100 \mathrm{~J}$, o equipamento protótipo reestabeleceu o ritmo sinusal em três suínos (75\%) e o equipamento referência realizou somente uma desfibrilação eficaz (25\%). A aplicação de energia $100 \mathrm{~J}$ associou-se com queimadura cutânea de Grau II em apenas um suíno com o desfibrilador protótipo e em quatro suínos com o equipamento referência. As lesões do tecido cardíaco ocorreram em igual número com ambos os desfibriladores.

Conclusões: O desfibrilador protótipo, quando aplicado com energia $100 \mathrm{~J}$, demonstrou maior eficácia na reversão da fibrilação ventricular e apresentou menor injúria que o desfibrilador de referência com a mesma energia. Este estudo ajudará a elevar o padrão tecnológico e a ampliar a oferta qualificada de equipamentos para a saúde produzidos no Brasil, substituindo e/ou diminuindo importações e oferecendo um dispositivo seguro de última geração.

DESCRITORES: FIBRILAÇÃO VENTRICULAR; EQUIPAMENTO; DESFIBRILADOR EXTERNO; SUÍNOS; INVESTIGAÇÃO PRÉ-CLÍNICA.

\section{ABSTRACT}

Aims: To evaluate the efficacy of defibrillation in response to induced ventricular fibrillation and to describe the macroscopic changes in porcine hearts after truncated exponential capacitive discharge shock applied by a prototype defibrillator produced in Brazil and a reference, international market leader defibrillator.

Methods: Sixteen pigs were divided into four groups of four animals each, to verify the effectiveness of the defibrillator in response to induced ventricular fibrillation by applying loads of $50 \mathrm{~J}$ to $100 \mathrm{~J}$, and assess the safety of the defibrillator by macroscopic analysis of the skin and heart. Erythema, edema, blistering, fluid in the pericardial cavity, burns, and post-shock charred tissue were evaluated.

Results: All pigs, after one minute of inducing ventricular fibrillation, developed tachycardia and progressed to ventricular fibrillation. When used low energy, the prototype equipment obtained $25 \%$ of success, with one reversed pig, while the reference equipment has obtained a success rate of $50 \%$, with two reversals. However, with energy $100 \mathrm{~J}$, the prototype equipment reestablished sinus rhythm in three pigs (75\%) and the reference product only made effective defibrillation in one (25\%). The application of $100 \mathrm{~J}$ energy was associated with skin burns Grade II in only one pig with the prototype defibrillator and four pigs with the reference equipment. The heart tissue injuries occurred in equal numbers with both defibrillators.

Conclusions: The prototype defibrillator, when applied with $100 \mathrm{~J}$ energy, was more effective in reversing ventricular fibrillation and showed less injury than the reference defibrillator with the same energy. This study may help to raise the technological standard and to increase the supply of qualified health equipment produced in Brazil, replacing and/or decreasing imports and providing a secure next-generation device. KEY WORDS: VENTRICULAR FIBRILLATION; EQUIPMENT; EXTERNAL DEFIBRILLATOR; SWINES; PRE-CLINICAL RESEARCH. 


\section{INTRODUÇÃO}

A área de desenvolvimento de equipamentos biomédicos no Brasil está crescendo com o apoio de programas governamentais e da iniciativa privada, pois há uma percepção pelo mercado e pelos órgãos do governo da necessidade de nacionalização de tecnologias e de investimentos em inovação. Tornase imprescindível que o país procure alcançar a suficiência tecnológica e industrial, garantindo sua inserção e permanência no mercado internacional, através do desenvolvimento de níveis de qualidade e competitividade. ${ }^{1}$ Com relação aos desfibriladores, estudos com objetivo de desenvolver e aprimorar esses equipamentos são importantes, devido à sua grande utilidade para a medicina contemporânea.

Existem poucas instituições que possuem o conhecimento necessário para projetar, testar e avaliar clinicamente dispositivos, identificar as causas de eventos adversos e mau funcionamento do equipamento, desenvolver projetos de dispositivos interativos e avaliar o processo de regulamentação. Para isso, há a necessidade de se trabalhar com a academia, a indústria e a comunidade de saúde com o intuito de desenvolver uma equipe multidisciplinar de profissionais, cobrindo as áreas de projeto de dispositivos e de engenharia, testes pré-clínicos, avaliação clínica, processos de regulação e monitoramento pós-comercialização. ${ }^{2}$

Nesse contexto, as caracterizações físicas de dispositivos médicos de desfibrilação, obtidas por meio de ensaios mecânicos e testes de segurança elétrica, constituem uma etapa crítica na transformação de conceitos inovadores e produtos confiáveis para o uso em seres humanos. Entretanto, a desfibrilação ventricular por meio de um choque elétrico não é necessariamente um procedimento inócuo; estudos têm demonstrado tanto dano morfológico quanto deficiência funcional do coração, causados por sucessivos choques. ${ }^{3-7}$ As informações obtidas por meio de ensaios pré-clínicos permitem relacionar segurança e eficácia do choque elétrico em animais experimentais e determinar quaisquer efeitos secundários indesejáveis em situações clínicas, de forma a avaliar se constituem riscos em função do funcionamento previsível do dispositivo. ${ }^{8} \mathrm{O}$ conhecimento dessas informações é fundamental para que a qualidade do produto seja assegurada e reconhecida.

Nesse trabalho, fruto de um projeto maior de uma parceira entre a Pontifícia Universidade Católica do Rio Grande do Sul (PUCRS), a Lifemed Indústria de Equipamentos e Artigos Médico-Hospitalares S.A e a Toth Tecnologia (TecnoPuc), financiado com o apoio da Finep - Inovação e Pesquisa, foram realizados estudos em modelo animal de comparação de choques de onda bifásica exponencial truncada num espectro de energia entre um protótipo operante de desfibrilador e outro referência, líder de mercado internacional.

Já está bem estabelecido que as ondas bifásicas são superiores tanto na eficácia de desfibrilação do primeiro choque ${ }^{9}$ rescisão de fibrilação ventricular, ${ }^{10}$ quanto na segurança, com menores riscos de disfunções miocárdicas e queimaduras na pele,${ }^{11} \mathrm{em}$ comparação às formas de ondas monofásicas. As formas de ondas bifásicas, assim, são utilizadas em quase todos os desfibriladores externos automáticos (DEA) vendidos atualmente, ${ }^{12}$ e são os mais produzidos pela indústria de equipamentos médicos, ${ }^{13}$ porém há dificuldades de se obter um consenso da melhor forma de onda bifásica (como exponencial truncada, retilínea, pulsada) a ser empregada. Nas diversas publicações patrocinadas por empresas fabricantes, ${ }^{14-16} \mathrm{e}$ mesmo entre os estudos não favorecidos, não há evidências de superioridades entre elas, ${ }^{17-19}$ pois existe uma falta de capacidade de toda onda bifásica lidar com diferentes impedâncias do ponto de vista elétrico. ${ }^{17}$

O objetivo deste estudo foi investigar o sucesso da cardiodesfibrilação em resposta à fibrilação ventricular induzida e avaliar as mudanças macroscópicas internas (pele) e externas (coração) de suínos após aplicação do choque pelos desfibriladores. Pretendeu-se também estudar a eficácia e a segurança do desfibrilador em desenvolvimento, de tecnologia totalmente nacional, com módulo de marca-passo, dotado de monitoração multiparamétrica e de um sistema de comunicação de dados sem fio. Acredita-se que este estudo ajudará a elevar o padrão tecnológico e a ampliar a oferta qualificada de equipamentos para a saúde produzidos no Brasil, substituindo e/ou diminuindo importações e oferecendo um dispositivo seguro de última geração.

\section{MÉTODOS}

Este estudo foi apreciado e aprovado pela Comissão de Ética no Uso de Animais (CEUA) da PUCRS através do registro de número CEUA 11/0023 e realizado no Laboratório de Habilidades Médicas da PUCRS.

Os desfibriladores externos utilizados nesta investigação foram o protótipo operante LifeSchock PRO (Lifemed, São Paulo) e o referência padrão ouro, líder de mercado, Philips HeartStart MRx, (Royal Philips, Amsterdã, Países Baixos) ambos com as mesmas características mecânicas.

Para avaliar eficácia da desfibrilação, considerouse a taxa de sucesso na reversão quando aplicadas 
cargas de $50 \mathrm{~J}$ e $100 \mathrm{~J}$, as quais foram estipuladas conforme as preconizadas pelas diretrizes da American Heart Association para uso pediátrico. ${ }^{12}$ Para avaliar a segurança do equipamento, realizaram-se avaliações macroscópicas externas (pele) e internas (coração) dos suínos pós-choque.

Foram utilizadas 16 dezesseis suínos fêmeas, mestiços das raças Landrace e Large White, pesando entre $20 \mathrm{~kg}$ e $30 \mathrm{~kg}$ (média \pm DP de $24,4 \pm 3,54 \mathrm{~kg}$ ), procedentes da granja Balduíno de Irmãos Johner em Cruzeiro do Sul, RS, certificada pelo Ministério da Agricultura, Pecuária e Abastecimento sob número 102/2012. Com base em consultoria estatística, os animais foram subdivididos em quatro grupos segundo: Grupo I: quatro animais, empregado o protótipo de equipamento a $50 \mathrm{~J}$ (PO-DEA $50 \mathrm{~J}$ ); Grupo II: quatro animais, empregado o protótipo de equipamento a $100 \mathrm{~J}$ (PO-DEA $100 \mathrm{~J})$; Grupo III: quatro animais, empregado o equipamento referência a $50 \mathrm{~J}$ (R-DEA 50J); e Grupo IV: quatro animais, empregado o equipamento referência a $100 \mathrm{~J}$ (R-DEA $100 \mathrm{~J}$ ) (Quadro 1).

Quadro 1. Distribuição dos suínos no estudo

\begin{tabular}{clc} 
Grupo & \multicolumn{1}{c}{ Desfibrilador } & $\begin{array}{c}\text { Número } \\
\text { de suínos }\end{array}$ \\
\hline I & Protótipo de equipamento a 50J (PO-DEA 50J) & 4 \\
II & Protótipo de equipamento a 100J (PO-DEA 100J) & 4 \\
III & Equipamento referência a 50J (R-DEA 50J) & 4 \\
IV & Equipamento referência a 100J (R-DEA 100J) & 4
\end{tabular}

A avaliação estatística para definição do número de animais para o estudo foi baseada em estudos publicados que envolvem o sistema cardiovascular, como o experimento de Walker et al., ${ }^{14}$ que avaliou a eficácia de seis formas de onda em episódio de fibrilação ventricular comparando choques monofásicos de $200 \mathrm{~J}$ e bifásicos de $150 \mathrm{~J}$ em grupos de seis e oito suínos, o de Li et al., ${ }^{16}$ onde foi estudada a eficácia das formas de ondas bifásicas pulsada e retilínea utilizando seis porcos domésticos, e o de Huang et al., ${ }^{20}$ que comparou os limiares de desfibrilação das formas de onda trifásica e bifásica em grupos de oito porcos. A ordem dos animais para a realização dos experimentos foi randomizada para os grupos e de forma cega para os laboratórios de análise. As amostras foram identificadas sequencialmente, sem que houvesse informações que pudessem ser tendenciosas para os analistas.

A técnica anestésica empregada pelo médico veterinário foi medicação pré-anestésica com quetamina $\mathrm{S}(+)\left(5 \mathrm{mg} \cdot \mathrm{kg}^{-1} \mathrm{Ketamin}^{\circledR}\right)$, midazolam $\left(0,3 \mathrm{mg} \cdot \mathrm{kg}^{-1}\right.$ Dormonid $\left.^{\circledR}\right)$ e sulfato de morfina $\left(0,5\right.$ mg. $\mathrm{kg}^{-1}$ Dolomorf $\left.^{\circledR}\right)$ via intramuscular. Com os animais sedados, realizou-se o acesso venoso na veia marginal da orelha e sua manutenção com solução de cloreto de sódio $0,9 \%$ (Baxter) a $5 \mathrm{ml} \cdot \mathrm{kg}^{-1} \cdot \mathrm{h}^{-1}$. Utilizando a mesma via, os animais foram submetidos à anestesia geral com propofol $\left(5 \mathrm{mg} \cdot \mathrm{kg}^{-1}\right.$ Propovan $\left.{ }^{\circledR}\right)$, colocados na posição esternalventral e intubados. A manutenção anestésica foi feita com propofol $\left(10 \mathrm{mg} \cdot \mathrm{kg}^{-1} \cdot \mathrm{h}^{-1}\right)$ e fentanil $\left(5 \mathrm{mcg} \cdot \mathrm{kg}^{-1} \cdot \mathrm{h}^{-1}\right.$ Fentanest $\left.^{\mathbb{R}}\right)$. Todo o procedimento foi feito sob o acompanhamento de médico veterinário. A ventilação foi espontânea, com os suínos recebendo oxigênio $100 \%$. O fentanil ( 2 mcg.kg ${ }^{-1} \cdot \mathrm{h}^{-1}{ }^{-}$Fentanest $\left.{ }^{\circledR}\right)$ foi utilizado quando necessária uma suplementação analgésica, no caso de alguma alteração relacionada à dor e sofrimento.

Para indução da fibrilação ventricular foi administrada solução de cloreto de potássio $(\mathrm{KCl} 10 \%)$ via intravenosa até a detecção da fibrilação. Para desfibrilação, foram utilizadas pás pediátricas que foram preparadas com gel condutor e posicionadas corretamente sobre o tórax dos suínos.

A análise estatística foi realizada por análise descritiva. Avaliações macroscópicas da pele foram feitas externamente para averiguar a presença de queimaduras e outras lesões cutâneas, classificando seus diferentes Graus (I, II, III). A presença de eritema/ vermelhidão e edema caracterizou queimadura de Grau I; a presença de tecido esbranquiçado e de bolhas, em queimadura de Grau II; e a presença de tecido carbonizado, visualização de tecido muscular e tecido ósseo, o Grau III. ${ }^{21}$

A avaliação de lesão pós-choque interna foi realizada macroscopicamente com auxílio do médico veterinário quanto à manifestação de eritema, edema, bolhas, líquido na cavidade pericárdica e tecido carbonizado nos corações retirados por uma incisão na linha do esterno.

A eutanásia dos animais foi realizada seguindo as condutas regulamentadas em leis estaduais e federais de ética na experimentação animal, sem proporcionar qualquer tipo de sofrimento, desconforto ou estresse aos suínos, de acordo com a Resolução $n^{\circ} 1000$, de 11 de maio de 2012 do Conselho Federal de Medicina Veterinária.

\section{RESULTADOS}

Com relação à indução da fibrilação ventricular, todos os suínos $(\mathrm{n}=16)$ após 1 minuto da aplicação de cloreto de potássio ( $\mathrm{KCl}$ a $10 \%)$, desenvolveram taquicardia, progredindo à fibrilação ventricular, que 
foi detectada em todos os eventos pelos desfibriladores.

Quanto ao sucesso na desfibrilação, o equipamento referência mostou-se mais eficaz na reversão da fibrilação ventricular quando utilizada baixa energia (Grupo III), com dois suínos revertidos (50\%), enquanto o equipamento protótipo obteve $25 \%$ de sucesso nesse nível de engergia usado para a desfibrilação (Grupo I). Contudo, com energia selecionada a $100 \mathrm{~J}$, o equipamento protótipo (Grupo II) reestabeleceu o ritmo sinusal em três suínos $(75 \%)$ e o equipamento referência realizou somente uma desfibrilação eficaz (Grupo IV) (Figura 1).

Quanto à existência de danos macroscópicos externos aparentes causados pela desfibrilação, 12 dos 16 suínos $(75 \%)$ apresentaram classificação de lesão Grau I.

Com relação às taxas de carga e equipamento utilizado, pôde-se observar que todos os animais do Grupo III (R-DEA 50 J) desenvolveram eritema e vermelhidão pós-choque, enquanto o uso do equipamento PO-DEA $50 \mathrm{~J}$ (Grupo I) resultou em eritema e vermelhidão em dois suínos e bolhas e tecido esbranquiçado em um animal (Figura 2).

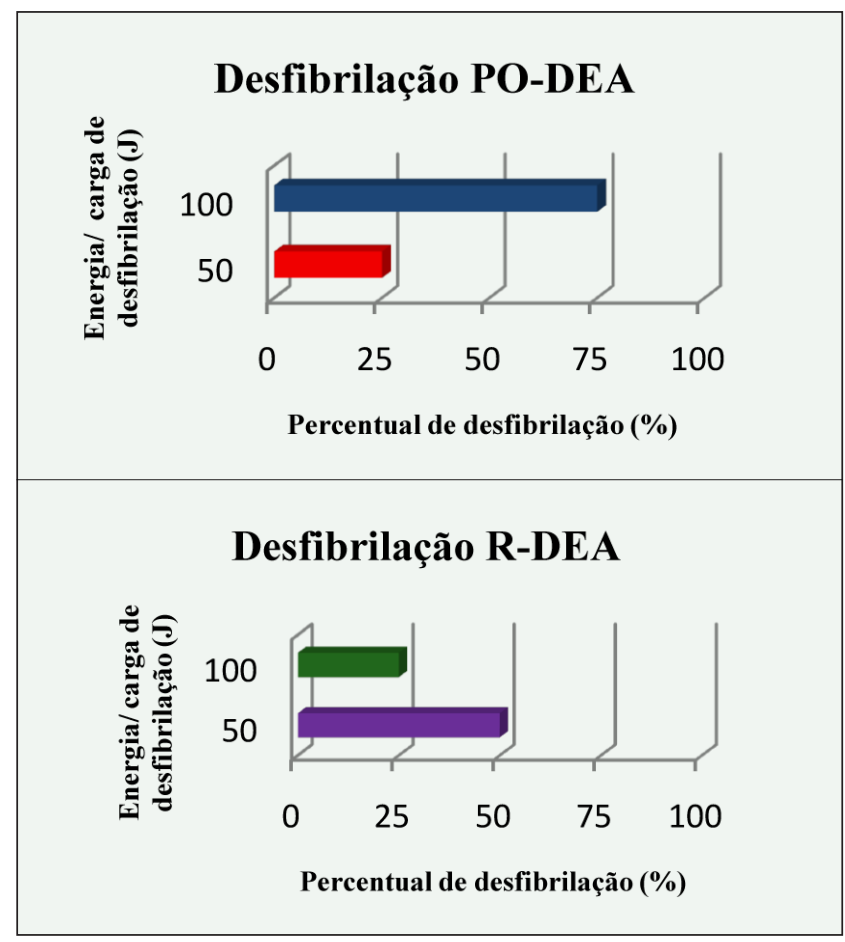

Figura 1. Percentual de desfibrilação do desfibrilador protótipo em estudo (PO-DEA) e do desfibrilador referência (R-DEA) nas energias 50 J e 100 J (O equipamento protótipo reverteu 75\% dos suínos com energia de $100 \mathrm{~J}$ e $25 \%$ com energia de $50 \mathrm{~J}$. O equipamento de referência reverteu $25 \%$ dos suínos com energia de 100 J e $50 \%$ dos suínos com energia de 50 J).

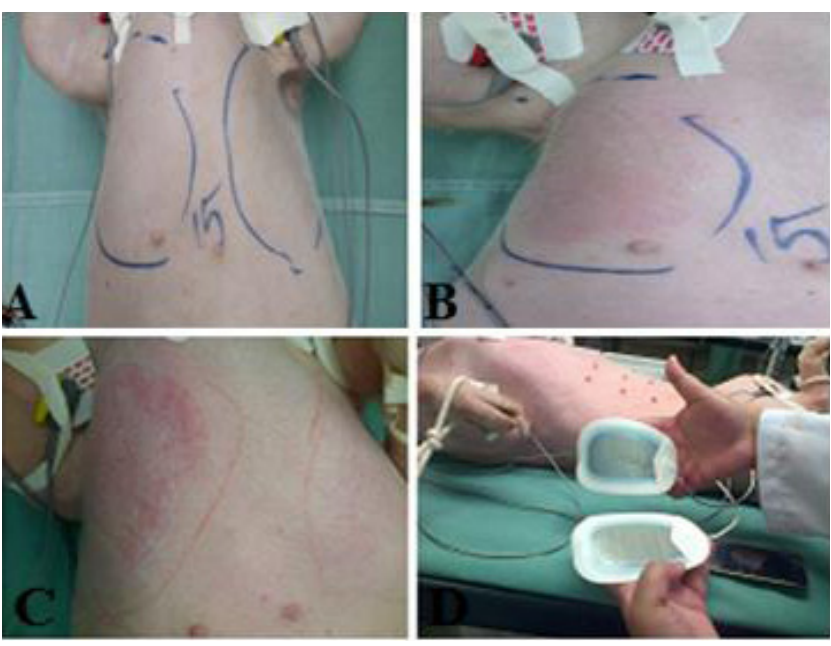

Figura 2. Lesão pós choque externa observada nos suínos. A: suíno 15 pré-choque. B: vermelhidão e eritema observado pós-choque no suíno 15. C: eritema e vermelhidão no suíno observado no local das pás. D: visualização das pás pediátricas.

O emprego do desfibrilador R-DEA com aplicação de energia $100 \mathrm{~J}$ (Grupo IV) associou-se com queimadura de Grau II em quatro suínos (bolhas e/ou tecido esbranquiçado). Nenhum suíno apresentou grau III de queimadura externa. Assim, quando na aplicação de carga $50 \mathrm{~J}$, o equipamento protótipo se mostrou menos seguro, pelo fato de um suíno apresentar grau II de queimadura (bolhas e tecido esbranquiçado). A Tabela 1 apresenta o resultado da análise macroscópica de lesão pós-choque externa com o número de animais que sofreram cada tipo de injúria.

Quanto à existência de lesão pós-choque interna, os resultados obtidos estão descritos na Tabela 2. Líquido na cavidade pericárdica ocorreu em três suínos em ambos os equipamentos nas cargas de $50 \mathrm{~J}$ (Grupo I e Grupo III) e em quatro suínos nas cargas de 100 J (Grupo II e Grupo IV). Edema foi encontrado em todos os corações $(100 \%)$ que sofreram desfibrilação com o equipamento protótipo, em contraponto ao equipamento referência, que apresentou $75 \%$ de frequência. Apenas um coração do Grupo IV apresentou, na região do átrio, presença de tecido carbonizado. Bolhas e eritema foram observados com maior incidência no equipamento referência.

Constatou-se que os algoritmos de detecção de taquiarritmias empregados em ambos os equipamentos foram eficazes após a aplicação do cloreto de potássio como indutor de fibrilação ventricular. Observou-se, todavia, grande variação no volume de $\mathrm{KCl}$ necessário para induzir a fibrilação de acordo com o peso de cada animal, que ficou compreendido entre $6-20 \mathrm{~mL}$, com uma média de $10 \mathrm{~mL} \pm 5,98 \mathrm{~mL}$ (Figura 3). 
Tabela 1. Resultado da análise macroscópica de lesão pós-choque externa

\begin{tabular}{|lccccc|} 
& PO-DEA 50 & PO-DEA 100 & R-DEA 50 J & R-DEA 100 J & Total \\
\hline $\begin{array}{l}\text { Grupo I } \\
\mathbf{n = 4}\end{array}$ & $\begin{array}{c}\text { Grupo II } \\
\mathbf{n = 4}\end{array}$ & $\begin{array}{c}\text { Grupo III } \\
\mathbf{n = 4}\end{array}$ & $\begin{array}{c}\text { Grupo IV } \\
\mathbf{n = 4}\end{array}$ & $\mathbf{n = 1 6}$ \\
\hline Eritema & $2(50 \%)$ & $3(75 \%)$ & $4(100 \%)$ & $3(75 \%)$ & $12(75 \%)$ \\
\hline Vermelhidão & $2(50 \%)$ & $3(75 \%)$ & $4(100 \%)$ & $3(75 \%)$ & $12(75 \%)$ \\
\hline Edema & $1(25 \%)$ & $1(25 \%)$ & $1(25 \%)$ & $1(25 \%)$ & $4(25 \%)$ \\
\hline Bolhas & $1(25 \%)$ & $0(0 \%)$ & $0(0 \%)$ & $1(25 \%)$ & $2(12,5 \%)$ \\
\hline Tecido esbranquiçado & $1(25 \%)$ & $1(25 \%)$ & $0(0 \%)$ & $3(75 \%)$ & $5(31,2 \%)$ \\
\hline Tecido carbonizado & $0(0 \%)$ & $0(0 \%)$ & $0(0 \%)$ & $0(0 \%)$ & $0(0 \%)$ \\
\hline Visualização de tecido muscular & $0(0 \%)$ & $0(0 \%)$ & $0(0 \%)$ & $0(0 \%)$ & $0(0 \%)$ \\
\hline Visualização de tecido ósseo & $0(0 \%)$ & $0(0 \%)$ & $0(0 \%)$ & $0(0 \%)$ & $0(0 \%)$ \\
\hline
\end{tabular}

PO-DEA 50J: desfibrilador protótipo em estudo, com energia $50 \mathrm{~J}$.

R-DEA 50J: desfibrilador referência, com energia 50 J.

PO-DEA 100J: desfibrilador protótipo em estudo, com energia 100J.

R-DEA 100J: desfibrilador referência, com energia 100 J.

Tabela 2. Resultado da análise macroscópica de lesão pós-choque interna

\begin{tabular}{lccccc} 
& PO-DEA 50J & PO-DEA 100J & R-DEA 50 J & R-DEA 100 J & Total \\
\hline Grupo I & $\begin{array}{c}\text { Grupo II } \\
\mathbf{n = 4}\end{array}$ & $\begin{array}{c}\text { Grupo III } \\
\mathbf{n = 4}\end{array}$ & $\begin{array}{c}\text { Grupo IV } \\
\mathbf{n = 4}\end{array}$ & $\mathbf{n = 1 6}$ \\
\hline Líquido na cavidade pericárdica & $3(75 \%)$ & $4(100 \%)$ & $3(75 \%)$ & $4(100 \%)$ & $14(87,5 \%)$ \\
\hline Tecido carbonizado & $0(0 \%)$ & $1(25 \%)$ & $0(0 \%)$ & $0(0 \%)$ & $1(0,62 \%)$ \\
\hline Edema & $4(100 \%)$ & $4(100 \%)$ & $3(75 \%)$ & $3(75 \%)$ & $14(87,5 \%)$ \\
\hline Bolhas & $3(75 \%)$ & $3(75 \%)$ & $3(75 \%)$ & $4(100 \%)$ & $13(8,12 \%)$ \\
\hline Eritema & $2(50 \%)$ & $3(75 \%)$ & $3(75 \%)$ & $4(100 \%)$ & $12(75 \%)$ \\
\hline
\end{tabular}

PO-DEA 50J: desfibrilador protótipo em estudo, com energia $50 \mathrm{~J}$.

R-DEA 50J: desfibrilador referência, com energia 50 J.

PO-DEA 100J: desfibrilador protótipo em estudo, com energia 100 J.

R-DEA 100J: desfibrilador referência, com energia $100 \mathrm{~J}$.

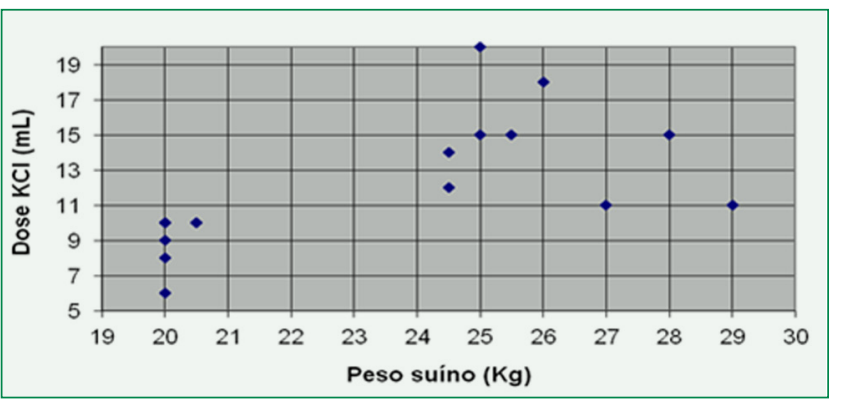

Figura 3. Relação dose cloreto de potássio $(\mathrm{KCl})$ versus peso do suíno, necessária para desencadear fibrilação ventricular

\section{DISCUSSÃO}

A grande variação no volume de $\mathrm{KCl}$ necessário para induzir a fibrilação demonstra as diferentes sensibilidades à ação do potássio no organismo, que podem ter sido influenciadas por diversos fatores como stress, doenças associadas, peso do animal, predisposições genéticas, entre outros.

$\mathrm{O}$ equipamento protótipo mostrou-se mais seguro que o de referência, quando aplicada a energia maior na avaliação de lesão pós-choque externa, associando-se apenas com edema na pele em $25 \%$ dos animais, (um suíno de cada grupo), enquanto ambos os equipamentos com taxa de carga $100 \mathrm{~J}$ causaram eritema e vermelhidão em $75 \%$ (três suínos) e presença de edema em $25 \%$ (um suíno). A disparidade na segurança dos equipamentos com essa energia foi caracterizada pela presença de tecido esbranquiçado em $75 \%$ (três suínos) do Grupo IV (R-DEA $100 \mathrm{~J}$ ), em contraponto com somente 25\% (um suíno) do Grupo II (PO-DEA 100 J).

É importante ressaltar que a avaliação da existência de lesão pós-choque interna foi realizada de forma subjetiva, exercida somente por avaliação macroscópica com auxílio do médico veterinário. Sugere-se que estudos de avaliação microscópica com ensaios bioquímicos com marcadores de lesão miocárdica e 
ensaios histológicos devam ser igualmente realizados para melhor embasamento dos resultados.

Foi possível estabelecer uma provável existência de comorbidade nos corações de alguns suínos; observou-se que dois corações dos suínos que sofreram choque de energia de $100 \mathrm{~J}$ apresentaram maior rigidez e entumecimento, e quatro suínos continham coágulos sanguíneos no seu interior. $\mathrm{O}$ único animal que não teve a fibrilação ventricular revertida no grupo PO-DEA $100 \mathrm{~J}$ foi o que apresentou tecido carbonizado na região do átrio.

Comparando os dados de eficácia na desfibrilação em resposta à fibrilação ventricular induzida e da segurança entre os desfibriladores protótipo e de referência por meio de alterações macroscópicas pósdesfibrilação, concluiu-se que o desfibrilador protótipo, quando aplicado com energia $100 \mathrm{~J}$, demonstrou melhor eficácia na rescisão da fibrilação ventricular e apresentou menor injúria que o desfibrilador de referência com a mesma energia.

Apesar deste estudo ser preliminar, os resultados observados são animadores e revelam a importância da inclusão da investigação pré-clínica para equipamentos que possam colocar em risco a vida do ser humano, pois mesmo a tecnologia mais sofisticada não consegue simular a complexidade das interações entre células, tecidos e órgãos. O prosseguimento do estudo, com um número amostral maior e seguindo com o mesmo rigor as normas de utilização de animais em pesquisas experimentais, poderá trazer resultados ainda mais conclusivos, tornando o novo equipamento apto para a inserção no mercado nacional e internacional.

\section{REFERÊNCIAS}

1. Instituto Brasileiro de Ensaios de Conformidade. Apresentação do laboratório de Segurança Elétrica - Ensaio de Inspeção, Elétricos e Mecânicos aplicados a Equipamentos Eletromédicos [Internet]. Hortolândia; 2012. [cited 2012 Dec 12]. Available from: http://www.ibec. com.br/download/Apostila_SegEletrica.pdf

2. Food and Drug Administration. Why are animals used for testing medical products [Internet]. Silver Spring: FDA; [2012]. [cited 2012 Dec 12]. Available from: http://www.fda.gov/AboutFDA/Transparency/Basics/ucm194932.htm

3. Babbs CF, Tacker WA, VanVleet JF, Bourland JD, Geddes LA. herapeutic indices for transchest defibrillator shocks: effective, damaging, and lethal electrical doses. Am Heart J. 1980 Jun;99(6):734-8.

4. Koumbourlis AC. Electrical injuries. Crit Care Med. 2002 Nov;30(11 Suppl):S424-30.

5. Ku CS, Lin SL, Hsu TL, Wang SP, Chang MS. Myocardial damage associated with electrical injury. Am Heart J. 1989 Sep;118(3):621-4.

6. Osswald S, Trouton TG, O’Nunain SS, Holden HB, Ruskin JN, Garan H. Relation between shock-related myocardial injury and defibrillation efficacy of monophasic and biphasic shocks in a canine model. Circulation. 1994 Nov;90(5):2501-9.

7. Dahl CF, Ewy GA, Warner ED, Thomas ED. Myocardial Necrosis from Direct Current Countershock: Effect of paddle electrode size and time interval between discharges. Circulation. 1974 Nov;50(5):956-61.

8. Maccarthy T. Animal models in medical device development and qualification. Charles River Laboratories.1997;10(2).

9. Higgins SL, Herre JM, Epstein AE, Greer GS, Friedman PL, Gleva ML, Porterfield JG, Chapman FW, Finkel ES, Schmitt PW, Nova RC, Greene HL. A comparison of biphasic and monophasic shocks for external defibrillation. Physio-Control Biphasic Investigators. Prehosp Emerg Care. 2000 Oct-Dec;4(4):305-13.

10. Mittal S, Ayati S, Stein KM, Knight BP, Morady F, Schwartzman D, Cavlovich D, Platia EV, Calkins H, Tchou PJ, Miller JM, Wharton JM, Sung RJ, Slotwiner DJ, Markowitz SM, Lerman BB. Comparison of a novel rectilinear biphasic waveform with a damped sine wave monophasic waveform for transthoracic ventricular defibrillation. ZOLL Investigators. J Am Coll Cardiol. 1999 Nov 1;34(5):1595-601.

11. Schwarz B, Bowdle TA, Jett GK, Mair P, Lindner KH, Aldea GS, Lazzara RG, O’Grady SG, Schmitt PW, Walker RG, Chapman FW, Tacker WA. Biphasic shocks compared with monophasic damped sine wave shocks for direct ventricular defibrillation during open heart surgery. Anesthesiology. 2003 May;98(5):1063-9.

12. Link MS, Atkins DL, Passman RS, Halperin HR, Samson RA, White RD, Cudnik MT, Berg MD, Kudenchuk PJ, Kerber RE. Part 6: electrical therapies: automated external defibrillators, defibrillation, cardioversion, and pacing: 2010 American Heart Association Guidelines for Cardiopulmonary Resuscitation and Emergency Cardiovascular Care. Circulation. 2010 Nov 2;122(18 Suppl 3):S706-19.

13. Abordagem de vigilância sanitária de produtos para saúde comercializados no Brasil: desfibrilador externo. Bol Inf Tecnovigilância [Internet]. 2011 Jan/Mar;(1). [cited 2012 Dec 12]. Available from: http://www.anvisa.gov.br/boletim_tecno/boletim_tecno_fev2011/ boletim tecno.html

14. Walker RG1, Melnick SB, Chapman FW, Walcott GP, Schmitt PW, Ideker RE. Comparison of six clinically used external defibrillators in swine. Resuscitation. 2003 Apr;57(1):73-83.

15. Mischke K, Schimpf T, Knackstedt C, Frechen D, Gramley F, Rana O, Saygili E, Meyer C, Kelm M, Schauerte P. Comparison of four clinically used external defibrillators for transesophageal defibrillation. Intensivmed. 2008;45(2):76-81.

16. Li Y, Wang H, Cho JH, Didon JP, Bisera J, Weil MH, Tang W. Comparison of efficacy of pulsed biphasic waveform and rectilinear biphasic waveform in a short ventricular fibrillation pig model. Resuscitation. $2009 \mathrm{Sept}$;00(9):1047-51.

17. Zelinka M, Buić D, Zelinka I. Comparison of five different defibrillators using recommended energy protocols. Resuscitation. 2007 Sept;74(3):500-7. 
18. Neal S1, Ngarmukos T, Lessard D, Rosenthal L. Comparison of the efficacy and safety of two biphasic defibrillator waveforms for the conversion of atrial fibrillation to sinus rhythm. Am J Cardiol. 2003 Oct 1;92(7):810-4.

19. Kim ML, Kim SG, Park DS, Gross JN, Ferrick KJ, Palma EC, Fisher JD. Comparison of rectilinear biphasic waveform energy versus truncated exponential biphasic waveform energy for transthoracic cardioversion of atrial fibrillation. Am J Cardiol. 2004 Dec 1;94(11): $1438-40$.

20. Huang J, KenKnight BH, Rollins DL, Smith WM, Ideker RE. Ventricular defibrillation with triphasic waveforms. Circulation. 2000 Mar 21;101(11):1324-8

21. Thibodeau GA, Patton KT. Estrutura e funções do corpo humano. Barueri: Manole; 2002.

22. Ambler JJ, Deakin CD. A randomised controlled trial of the effect of biphasic or monophasic waveform on the incidence and severity of cutaneous burns following external direct current cardioversion. Resuscitation. 2006 Dec;71(3):293-300. 